\title{
Human Immunodeficiency Virus type 1 Drug Resistance Mutations in Patients Failing Antiretroviral Therapy in Lebanon from 2009 to 2013
}

\author{
Ahmad A. Hachem ${ }^{19}$, Essa H. Hariri" ${ }^{19}$, Anthony Mansour ${ }^{19}$, Jacques Mokhbat ${ }^{1,2}$ \\ ${ }^{1}$ School of Medicine, Lebanese American University, Byblos, Lebanon \\ ${ }^{2}$ Department of Internal Medicine, Lebanese American University Medical Center - Rizk Hospital, Beirut, \\ Lebanon \\ "These authors contributed equally to this work
}

Corresponding Author: Jacques Mokhbat, Beirut, Lebanon, jacques.mokhbat@gmail.com

Financial support: None

Conflict of Interest: None

Consent: Written consent was taken from the participants for the publication of this study

\section{Abstract}

Background: Antiretroviral drug resistance remains a significant problem in the clinical management of patients infected with the Human Immunodeficiency Virus type-1.

Aim: This study investigates and reports data on the molecular characterization of HIV-1 isolates from patients who are in a state of therapy failure.

Methods: This is a retrospective study conducted on 65 patients in therapy failure. Inclusion criteria included patients diagnosed as being in therapy failure between the years 2009 and 2013. We defined ART failure as either a failure to achieve viral suppression or a failure to detect viral loads below 500 copies $/ \mathrm{mL}$ after virological suppression in at least two plasma samples. We used the published WHO list for surveillance of transmitted resistance and the Stanford HIV Drug Resistance Database to identify drug resistance mutations.

Results: $65 \%$ of the participants had at least one drug resistance mutation (DRM). $12 \%$ of the population sampled had resistance to only one ART class, 32\% presented with resistance to two classes of antiretroviral drugs, and $20 \%$ had resistance to all three classes of drugs. The prevalence of nucleoside transcriptase inhibitor (NRTI) mutations was 55\%, the most common DRM being M184V. The prevalence of non-nucleoside reverse transcriptase inhibitor (NNRTI) mutations was 58\%, with the most common mutation being the $\mathrm{K} 103 \mathrm{~N}$ mutation. The prevalence of protease inhibitors drug resistance mutations was $23 \%$, with mutations V82A and I47V being present in $10 \%$ of the study population.

Conclusion: Our study is the first molecular characterization of DRM emergence in HIV-1 strains from patients failing antiretroviral therapy in Lebanon. Continuous monitoring of resistance patterns for HIV in the country is necessary to tackle the emergent drug resistance.

Keywords: HIV-1, ART, ART failure, resistance mutations, NRTIs, NNRTIs, PIs, Lebanon 


\section{Introduction}

Antiretroviral resistance is a significant issue in public health that has undermined the efficacy of antiretroviral treatment [1]. This resistance is mainly due to gene mutations selected for during suboptimal treatment periods, allowing for the emergence of new strains with decreased susceptibility to antiretroviral therapy [1, 2]. Different factors may lead to such mutations: large genetic variation of the virus, poor compliance with antiretroviral treatment, and sometimes drug therapy that may select for specific mutations. Although combination regimens have decreased the rate of mutations, the ability to prevent multi-regimens failure is still poorly defined [2]. Prevalence studies report failure rates of dual antiretroviral therapy as high as $60 \%[3,4]$. Hence, the continuous rise in resistance rates and failure of response to treatment has prompted a worldwide effort to follow, map, and monitor antiretroviral mutations in order to control this problem.

Regarding mapping mutations, we differentiate between 'treatment naïve' patients, who have not started therapy but have been infected with HIV, and patients 'failing therapy,' who have not responded to first-line treatment [1]. In Lebanon, since the first case diagnosed in 1984 [5], the prevalence of HIV has been $0.1 \%$ of the population. The reported cases of HIV/AIDS in 2010 in Lebanon were 1,346, according to UNAIDS [6]. The use of ART started with zidovudine in 1988 as a monotherapy, followed by didanosine in 1991. In 1996, zidovudine and didanosine (ddl) were combined, the latter being replaced by lamivudine (3TC). Indinavir was also being used at the time. Stavudine was introduced in the late 1990s, followed by efavirenz in the 2000s [7].

In Lebanon, HIV-infected patients receive their treatment free of charge from the National AIDS Program (NAP) and the Lebanese Ministry of Public Health (MOPH). The management and treatment of HIV patients in Lebanon follows the WHO recommendations [8]. The first-line treatment consists of two NRTIs: lamivudine (3TC) and zidovudine (AZT/ZDV), or tenofovir (TDF) and an NNRTI like efavirenz (EFV) and, less commonly, nevirapine (NVP). The second line treatment consists of two NRTIs plus a protease inhibitor: lopinavir/ritonavir (or ritonavir 'boosted'). The current antiretrovirals available for HIV-infected patients in Lebanon are zidovudine (AZT/ZDV), lamivudine (3TC), didanosine (ddl), tenofovir (TDF), abacavir (ABC) (all of which are NRTIs), efavirenz (EFV), and nevirapine (NVP) (both being NNRTIs), one protease inhibitor lopinavir/ritonavir (LPVr) and finally one integrase inhibitor (raltegravir RAL) [7]. We do not commonly use other drug classes such as fusion inhibitors and CCR5 antagonists in Lebanon. However, there are frequent shortages of drugs associated with increased resistance, which further complicates national efforts to monitor resistance patterns [9]. This study aims to present data on the molecular characterization of HIV-1 isolates from patients who are in a state of therapy failure.

\section{Methods}

\section{a) Study Participants}

Sixty-five HIV-1 infected patients, currently followed at the Lebanese American University Medical Center - Rizk Hospital (LAUMC-RH), provided written formal consent upon enrollment in the study. Human subject approval for this study was obtained from the Institutional Review Board (IRB) of the Lebanese American University Medical Center - Rizk Hospital (LAUMC-RH). Inclusion criteria included patients diagnosed as being in therapy failure between the years 2009 and 2013. ART failure was defined either as a failure to achieve virological suppression or as a failure to detect, in two plasma samples, viral loads below 500 copies $/ \mathrm{mL}$ after virological suppression. The current treatment for HIV includes three types of drugs: protease inhibitors, nucleoside and nucleotide reverse transcriptase inhibitors (NRTIs), and nonnucleotide reverse transcriptase inhibitors (NNRTIs).

\section{b) Clinical and Virologic Characteristics}

Blood samples were taken from all subjects for RNA extraction using the TRUGEN BAYER kit. Plasma was collected from patients with a volume of $1 \mathrm{~mL}$. The samples were then centrifuged at a speed of $28,000 \mathrm{~g}$ for 60 minutes at a temperature of $4^{\circ} \mathrm{C}$. HIV RNA concentrations of 50 to 1,000 copies $/ \mathrm{mL}$ were then obtained. We removed $860 \mu \mathrm{L}$ of supernatant. The resultant pellet was then resuspended. We utilized the TruGene HIV-1 genotyping assay which includes 
a kit for a reverse-transcriptase polymerase chain reaction (RT-PCR) for HIV RNA extracted from plasma. We did PCR amplification and cycle sequencing, specifically of the 1,318-bp fragment of the pol gene. This generates sequences for two genes: (a) the PR gene (288bp sequence; codons 4 to 99) and (b) the RT gene (630-bp sequence; codons 36 to 247). Finally, we analyzed the data, generated the gene sequences and their corresponding translated protein, and assessed the presence of antiretroviral resistance-conferring polymorphism using the OpenGene (Bayer Diagnostics) software system [10].

\section{c) HIV-1 Drug Resistance Mutations}

We used the published WHO list for surveillance of transmitted resistance and the Stanford HIV Drug Resistance Database [12] to identify drug resistance mutations [11-13]. According to the Stanford HIV database, each mutation is given a drug penalty score determining the level of resistance to that particular drug. Scores are attributed as the following: Score 0-9: susceptible to the drug, score 10-14: potential low-level resistance to the drug, score 15-29: low-level resistance to the drug, score 30-59: Intermediate-level resistance to the drug and finally a score above 60 entails high-level resistance to the particular drug. For the purpose of our study, drug resistance mutations (DRM) were defined as those having a Stanford score of a minimum of 30 (Stanford 30).

\section{d) Statistical Analysis}

We used descriptive statistics to characterize the mutation frequencies, percentages, and distribution, based on the drug type. We used tables to present the genetic mutations found in the recruited cohort from the Lebanese population. We carried out all analyses using STAT MP 13.0.

\section{Results}

Among the 65 patients recruited to the study, 9 (13\%) were in ARV failure in 2009, 7 (10\%) failed in 2010, 19 patients (27\%) failed in 2011, 9 (13\%) in 2012 and 21 (37\%) in 2013. The subjects' mean age was $40.88+/-6.6$ years, and most of them $(n=56,83.58 \%)$ were males. All patients were on two NRTIs: zidovudine (AZT/ZDV) and lamivudine (3TC). Figure 1 shows the distribution of the classes of mutations. NRTI and NNRTI resistance were the most commonly observed amongst ART failing patients; we detected NRTI Drug Resistance Mutations (DRMs) in 36 patients (55\%) and NNRTI DRMs in 38 patients (58\%). PI resistance was detected in 15 patients (23\%). 42 patients (64\%) presented with at least one DRM. Regarding classes of antiretrovirals, 8 patients (12\%) were resistant to only one class of ART, 21 patients (32\%) were resistant to two classes of antiretrovirals, and 13 patients (20\%) were resistant to all three classes of ART drugs. 23 patients $(35 \%)$ presented with no DRM that would explain their therapy failure. Table 1 summarizes genotypic resistance to antiretroviral drugs in subjects on therapy.

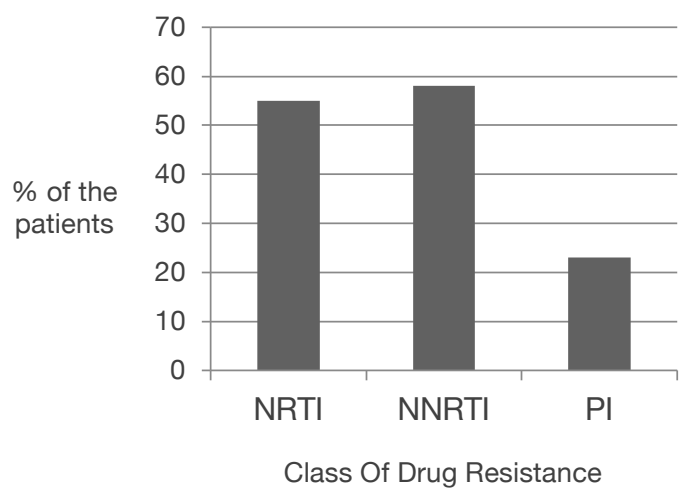

Figure 1: Drug resistance mutations by class in the study participants. NNRTI: non-nucleoside reverse transcriptase inhibitor; NRTI: nucleoside reverse transcriptase inhibitor; PI: protease inhibitor.

Table 1: Genotypic resistance to antiretroviral drugs in subjects failing therapy

\begin{tabular}{ll} 
Category & $\begin{array}{l}\text { Number of treated } \\
\text { showing resistance (\%) }\end{array}$ \\
\hline Overall & $42(64)$ \\
\hline NRTI & $36(55)$ \\
NNRTI & $38(58)$ \\
PI & $15(23)$
\end{tabular}

Two drug families $21(32)$

All three drug families $13(20)$ 
Regarding NRTIs, the most common mutation was the M184V detected in 30 patients (46\% of participants), followed by T215YF in 21 patients (32\% of participants), K70R in 8 patients (12\%), V75M in 5 patients $(7.6 \%)$ and $\mathrm{K} 65 \mathrm{R}$ in 4 patients (6.1\%). M184V is a signature mutation for lamivudine (3TC) and emtricitabine (Stanford 60 for both drugs) [14]. Its presence is not a contraindication to continued treatment with 3TC/FTC because of the increased susceptibility to AZT, TDF, and d4T (Stanford -10) [7, 15, 16]. Moreover, patients with the M184V mutation and on therapy have shown a clinically significant reduction in HIV-1 viral loads [17]. M184V may also increase susceptibility in patients who have already developed resistance to Zidovudine [18]. Another mutation with this particular characteristic is the $\mathrm{K} 65 \mathrm{R}$ mutation, which we found in 4 participants. K65R causes high-level resistance to TDF, ddl (Stanford 60), and intermediate-level resistance to ABC (Stanford 45) and 3TC(Stanford 30) [13]. On the other hand, it has also been shown to increase susceptibility to AZT (Stanford -15) [19]. K65R and M184V were conjointly found in 2 of the participants. Another primary NRTI mutation reported in the HIV database was the T215 Y/F found in 21 participants. This is a thymidine analog mutation causing intermediate to high-level resistance for AZT, d4T (Stanford 45 score), and low-level resistance to $A B C$, ddl, and TDF. The K70R mutation causes intermediate-level resistance to AZT (Stanford score of 30 ) and has low-level resistance for d4T, ABC, and ddl $[18,20]$.

Six major NNRTI resistance-conferring mutations were detected in our population sample. The most common of these mutations was the $\mathrm{K} 103 \mathrm{~N}$ mutation detected in 17 patients (26.1\%), followed by G190 A/S/V in 15 patients (23\%), Y181C in 9 patients (13\%), $\mathrm{P} 225 \mathrm{H}$ in 8 patients (12\%), Y188L in 3 patients $(4 \%)$ and finally K101E, which was detected in only one patient. K103N and $\mathrm{Y} 181 \mathrm{C}$ are known to confer cross-resistance to all NNRTIs. K103N causes high-level resistance to NVP with more than a 50fold reduction in drug susceptibility [21]. It also equally affects EFV, and the mutation has a Stanford score of 60 for both drugs [13, 22]. Y181C also severely affects susceptibility to NVP and EFV (Stanford 60 for NVP and Stanford 30 for EFV) $[12,13,23]$. G190A/S/V confers highlevel resistance to NVP (Stanford 60) with more than a 50 -fold reduction in susceptibility to the drug. It also confers intermediate-level resistance to EFV (Stanford 45) with a 5 to 10 fold decrease in drug susceptibility [12, 24, 25]. $\mathrm{P} 225 \mathrm{H}$ also causes intermediate-level resistance to EFV and NVP (both Stanford 30 scores). $\mathrm{P} 225 \mathrm{H}$ occurred with $\mathrm{K} 103 \mathrm{~N}$ in 3 of our participants. This specific combination of mutations causes high-level resistance to EFV, particularly with more than 50 fold reduction in susceptibility to the Efavirenz $\operatorname{EFV}[13,20]$.

Almost all patients presenting with at least one DRM showed a mutation in the protease gene (90\% of the patients, $n=60)$. The most common mutations throughout the years were the L63P and M36I mutations (cumulative percentages of $82 \%$ and $63 \%$ for L63 and M36, respectively). According to the Stanford HIV database [11, 13], L63P and M36I mutations are neither major nor minor resistance mutations. They confer no resistance to lopinavir, which Lebanese HIV patients have as part of their tri-therapy regimen. Amongst the PI DRM detected in the sample population, the most common mutations reported as major drug resistance mutations by the HIV Drug Resistance Database and the updated surveillance list of drug resistance mutations were V82A and I47V in 7 patients each (10\% each), followed by L9OM in 5 patients (7.7\%), I84V in 3 patients (4.6\%) and V32I, which was detected in only 2 of our patients. $184 \mathrm{~V}$ is associated with high-level resistance to ATV, FPV, IDV, NFV, SQV (all Stanford 60 scores), intermediate-level resistance to $\mathrm{LPV} / \mathrm{r}$, ritonavirboosted, and TPV/r (Stanford 30). L90M reduces susceptibility to all Pls except TPV and DRV (Stanford 60 for NFV and Stanford 40 for SQV). The two most common DRMs, V82A and I47V, are both associated with intermediate-level resistance (Stanford 30) for IDV, LPV/r, and NFV (for V82A) and FPV/r, TPV/r (for I47V) [13, 26, 27]. Minor PI mutations were found in one subject in our study (Stanford scores less than 20), namely in the I54V and M46I genes. The mutation I54V reduces susceptibly to all Pls except $D R V / r$ with mutation scores of Stanford 20. M46I reduces susceptibility to IDV, NFV, LPV, and ATV with a Stanford score of 10 (potential low-level resistance) $[12,20]$.

\section{Discussion}

This patient population represents a serious threat to the spread of the drug-resistant virus. 
This is consistent with the data of higher rates of transmission of drug resistant HIV from 2007 to 2013. This transmitted resistance causes impairment of treatment responses and highlights the urgent need for prevention efforts directed at this specific group of patients [7, 28].

In this study, $64 \%$ of the patients presented with at least one DRM. Regarding antiretroviral classes, $12 \%$ of the patients were resistant to only one ART class, $32 \%$ were resistant to two classes of antiretrovirals, and $20 \%$ were resistant to all three classes of drugs. In comparison, other countries such as South Africa [4], Brazil [29], France [30], and Spain [31] noted the existence of at least one DRM in $71 \%$ to $83 \%$ of the sequences, and resistance to two drug classes was between $48 \%$ to $58 \%$. Resistance to three classes was between $13 \%$ and $20 \%$. Also, 35\% of the patients showed no drug resistance mutations despite ART failure, whereas other studies demonstrated lower prevalence (14\% to $22 \%)$ for such a finding [2, 30-32]. This is probably due to inadequate adherence to therapy [31] and Lebanon's drug shortage episodes [7].

The high prevalence of NRTI and NNRTI DRMs (55\% for NRTI and $58 \%$ for NNRTIs) can be explained by the fact that a combination of NRTI and NNRTI is the first-line therapy for all patients. $[28,33]$. NRTI mutation prevalence in Lebanon amongst treatment failing patients $(55 \%)$ is closer to prevalence figures in Morocco (48\%) but far lower than prevalence figures in the United States, Spain, France, and England, where resistance to NRTI ranged between $71 \%$ $82 \%(28,30,31,33)$. The predominant NRTI mutation was $\mathrm{M} 184 \mathrm{~V}$, and its prevalence (46\%) was lower than described for HIV patients failing HAART in other countries: $70 \%$ prevalence in Morocco, $75 \%$ in Brazil, and $64.3 \%$ in South Africa, and 49.3\% in Spain [4, 28, 29, 34].

This substantially high prevalence in Lebanon may primarily be due to the extensive use of lamivudine (3TC). Furthermore, Lebanon is facing a net decrease in the incidence of M184V. This is most obvious when the 2013 incidence is compared to that of preceding years: $85.7 \%$ in $2010,47.4 \%$ in $2011,67 \%$ in 2012 , followed by a drop as low as $23.5 \%$ in 2013 [6]. Before 2013, M184V was the single most common NRTI mutation every year. This trend goes exactly with worldwide observations of $\mathrm{M} 184 \mathrm{~V} / \mathrm{I}$ mutation frequencies, which have been decreasing significantly over time. The increased use of a tenofovir/emtricitabine/efavirenz single-tablet regimen was associated with a reduced selection of these mutations (32). Lebanon began implementing the single-tablet regimen in 2009 , which correlates with the findings of the study. Prevalence of NNRTIs mutation (58\%) is slightly higher than that reported in other studies, ranging from $38.9 \%$ in France to $52 \%$ in England $[28,30,31,33]$. However, this also goes with the fact that NNRTIs are part of first-line therapy in ART. The most common NNRTI resistance mutation was $\mathrm{K} 103 \mathrm{~N}$, and this corresponds to other international figures as well $[2,35,36]$.

Regarding PI DRMs, the most prevalent DRMs in the study were the V82A and $147 \mathrm{~V}$ mutations. This contrasts with results worldwide regarding protease inhibitor mutations, where L90M, V82A $\mathrm{M} 46 \mathrm{l}$ are the most common. These are major resistance-conferring mutations [20, 35-37]. The prevalence of PI DRM in the study was $21.5 \%$, which is somewhat similar to prevalence figures in Morocco, but extensively much lower than those reported in Spain, France, Italy, and the United States, where PI DRM occurred in $41 \%$ to $53 \%$ of samples analyzed [28-34].

The genotypic analysis may be of crucial importance in guiding the clinician during management. For example, for NRTI, each drug has a predictable set of mutations beginning with primary mutations that decrease viral susceptibility to the drug. On the other hand, the disadvantage is a reduction in viral fitness [18] and the replication ability of the virus. Thus, a primary mutation is followed by a secondary mutation, which increases viral fitness. Detecting such primary mutations, which decrease viral fitness before the secondary mutations can form, can be beneficial in ART management as the clinician can adapt the therapeutic approach to such findings and curb the predictable progress of mutation-selection by exploiting this low level of viral replication [38]. This can have a significant impact on decreasing resistance to current ART regimens. Furthermore, infection with HIV strains with DRM will condemn the treatment-naïve patient to therapy failure earlier on in the course of treatment; thus, sequencing these strains presents as an increasingly unavoidable recommendation in the worldwide effort to 
decrease ART resistance in the clinical management of HIV. The International AIDS Society USA guidelines for the use of ART in adults advise testing for HIV drug resistance before starting therapy [39].

\section{Conclusion}

Our study shows a significantly high prevalence of drug resistance mutations among our study population, consisting of patients failing standard ART. Advances in HIV prevention and treatment with newer antiretroviral agents such as fusion inhibitors, integrase inhibitors and CCR5 inhibitors has significantly improved the survival and quality of life of patients with HIV.

With continued development of drugs and combination therapies, HIV resistance testing becomes even more important to tackle this emergent drug resistance and incorporate the regular use of pre-treatment testing for a sustainable adjustment of population-specific HIV treatments. This may potentially change what is considered "first-line".

\section{References}

1. Deeks SG, Gange SJ, Kitahata MM, et al. Trends in multidrug treatment failure and subsequent mortality among antiretroviral therapy-experienced patients with HIV infection in North America. Clin Infect Dis. 2009;49(10):1582-1590. PMid:19845473 PMCid:PMC2871149. https://doi.org/10.1086/644768

2. Couto-Fernandez JC, Silva-de-Jesus C, Veloso VG, et al. Human immunodeficiency virus type 1 (HIV-1) genotyping in Rio de Janeiro, Brazil: assessing subtype and drug-resistance associated mutations in HIV-1 infected individuals failing highly active antiretroviral therapy. Mem Inst Oswaldo Cruz. 2005; 100(1):73-78. PMid:15867968. https://doi.org/10.1590/s0074-02762005000100014

3. Jaka HM, Mshana SE, Liwa AC, et al. Prevalence of immunological failure and durability of first line antiretroviral therapy at Bugando Hospital Mwanza, Tanzania. Tanzania Medical Journal. 2009;24(2). https://doi.org/10.4314/tmj.v24i2.53278

4. Marconi VC, Sunpath H, Lu Z, et al. Prevalence of HIV-1 drug resistance after failure of a first highly active antiretroviral therapy regimen in KwaZulu Natal, South Africa. Clin Infect Dis. 2008;46(10):1589-1597. Expert Rev Anti Infect Ther. 2004;2(1):147-151. PMid:15482179.https://doi.org/10.1586/14787210.2. 1.147
5. Mokhbat JE, Ibrahim NK, Abdul-Karim FW, et al. The acquired immunodeficiency syndrome (AIDS): report of the first case in Lebanon and a review of the literature. Lebanese Medical Journal. 1985;35(4):295319.

6. UNAIDS. Global Aids Update 2020 [Available from: https://www.unaids.org/en/regionscountries/countrie s/lebanon.

7. Mokhbat JM, Melhem NM, El-Khatib Z, Zalloua P. Screening for antiretroviral drug resistance among treatment-naive human immunodeficiency virus type 1-infected individuals in Lebanon. J Infect Dev Ctries. 2014;8(3):339-348. Published 2014 Mar 13. PMid:24619266. https://doi.org/10.3855/jidc.3593

8. WHO. Antiretroviral therapy for HIV infection in adults and adolescents: recommendations for a public health approach. 2010.

9. Imaz A, Olmo M, Peñaranda M, et al. Short-term and long-term clinical and immunological consequences of stopping antiretroviral therapy in HIV-infected patients with preserved immune function. Antivir Ther. 2013;18(1):125-130. PMid:22805174. https://doi.org/10.3851/IMP2249

10. Kuritzkes DR, Grant RM, Feorino $P$, et al. Performance characteristics of the TRUGENE HIV-1 Genotyping Kit and the Opengene DNA Sequencing System. J Clin Microbiol. 2003;41(4):1594-1599. PMid:12682150 PMCid:PMC153857. https://doi.org/10.1128/jcm.41.4.1594-1599.2003

11. Bennett DE, Camacho RJ, Otelea D, et al. Drug resistance mutations for surveillance of transmitted HIV-1 drug-resistance: 2009 update. PLoS One. 2009;4(3):e4724.

PMid:19266092

PMCid:PMC2648874

https://doi.org/10.1371/journal.pone.0004724

12. Liu TF, Shafer RW. Web resources for HIV type 1 genotypic-resistance test interpretation. Clin Infect Dis. 2006;42(11):1608-1618. PMid:16652319 PMCid:PMC2547473 https://doi.org/10.1086/503914

13. Gifford RJ, Liu TF, Rhee SY, et al. The calibrated population resistance tool: standardized genotypic estimation of transmitted HIV-1 drug resistance. Bioinformatics. 2009;25(9):1197-1198. PMid:19304876 PMCid:PMC2672634 https://doi.org/10.1093/bioinformatics/btp134

14. Kasang C, Kalluvya S, Majinge C, et al. HIV drug resistance (HIVDR) in antiretroviral therapy-naïve patients in Tanzania not eligible for WHO threshold HIVDR survey is dramatically high. PLoS One. 2011;6(8):e23091.

PMid:21886779.

PMCid:PMC3158766.

https://doi.org/10.1371/journal.pone.0023091 
15. Paredes R, Clotet B. Clinical management of HIV1 resistance. Antiviral Res. 2010;85(1):245-265. PMid:19808056.

https://doi.org/10.1016/j.antiviral.2009.09.01516

16. Wainberg MA. The impact of the M184V substitution on drug resistance and viral fitness. Expert Rev Anti Infect Ther. 2004;2(1):147-151. PMid:15482179.

https://doi.org/10.1586/14787210.2.1.147

17. Turner D, Brenner B, Wainberg MA. Multiple effects of the M184V resistance mutation in the reverse transcriptase of human immunodeficiency virus type 1. Clin Diagn Lab Immunol. 2003;10(6):979981. PMid:14607855 PMCid:PMC262455. https://doi.org/10.1128/cdli.10.6.979-981.2003

18. Loveday C. International perspectives on antiretroviral resistance. Nucleoside reverse transcriptase inhibitor resistance. J Acquir Immune Defic Syndr. 2001;26 Suppl 1:S10-S24. PMid:11264998. https://doi.org/10.1097/00042560200103011-00003

19. Winston A, Stebbing J. The K65R mutation in HIV1 reverse transcriptase. J HIV Ther. 2004;9(2):25-27.

20. Rhee SY, Liu T, Ravela J, Gonzales MJ, Shafer RW. Distribution of human immunodeficiency virus type 1 protease and reverse transcriptase mutation patterns in 4,183 persons undergoing genotypic resistance testing. Antimicrob Agents Chemother. 2004;48(8):3122-3126. PMid:15273130 PMCid:PMC478552.

https://doi.org/10.1128/AAC.48.8.3122-3126.2004

21. Wang $Y$, Xing $H$, Liao L, et al. The development of drug resistance mutations K103N Y181C and G190A in long term Nevirapine-containing antiviral therapy. AIDS Res Ther. 2014;11:36. Published 2014 Nov 21. PMid:25926857

PMCid:PMC4414387. https://doi.org/10.1186/1742-6405-11-36

22. Quiñones-Mateu ME, Arts EJ. Fitness of drug resistant HIV-1: methodology and clinical implications. Drug Resist Updat. 2002;5(6):224-233. https://doi.org/10.1016/s1368-7646(02)00123-1

23. Vandamme AM, Sönnerborg A, Ait-Khaled M, et al. Updated European recommendations for the clinical use of HIV drug resistance testing. Antivir Ther. 2004;9(6):829-848.

24. Peeters M, Nijs S, Vingerhoets J, Tambuyzer L, et al. Determination of phenotypic clinical cut-offs for etravirine: pooled week 24 results of the DUET-1 and DUET-2 trials. Workshop, June 10-14, 2008, Sitges, Spain. XVII HIV Drug Resistance Antiviral therapy. 2008;13(Suppl. 3):A133.

25. Vingerhoets J, Peeters M, Azijn H, Tambuyzer L, et al. An update on the list of NNRTI mutations associated with decreased virological response to etravirine: multivariate analysis on the pooled DUET-1 and DUET-2 clinical trial data. XVII HIV Drug Resistance Workshop, June 10-14, 2008, Sitges, Spain. . Antiviral therapy. 2008;13(Suppl. 3, A134 (abstract 24).

26. Condra JH, Schleif WA, Blahy OM, et al. In vivo emergence of HIV-1 variants resistant to multiple protease inhibitors. Nature. 1995;374(6522):569-571. PMid:7700387. https://doi.org/10.1038/374569a0

27. Zhang YM, Imamichi H, Imamichi T, et al. Drug resistance during indinavir therapy is caused by mutations in the protease gene and in its Gag substrate cleavage sites. J Virol. 1997;71(9):66626670. PMid:9261388 PMCid:PMC191944. https://doi.org/10.1128/JVI.71.9.6662-6670.1997

28. Richman DD, Morton SC, Wrin T, et al. The prevalence of antiretroviral drug resistance in the United States. AIDS. 2004;18(10):1393-1401. PMid:15199315.https://doi.org/10.1097/01.aids.0000 131310.52526.c7

29. Toledo PV, Carvalho DS, Romagnoli L, et al. HIV-1 genotypic resistance profile of patients failing antiretroviral therapy in Paraná, Brazil. Braz J Infect Dis. 2010;14(4):360-371. https://doi.org/10.1590/S1413-86702010000400009 https://doi.org/10.1016/S1413-8670(10)70076-3

30. Tamalet C, Fantini J, Tourres C, Yahi N. Resistance of HIV-1 to multiple antiretroviral drugs in France: a 6year survey (1997-2002) based on an analysis of over 7000 genotypes. AIDS. 2003;17(16):2383-2388. PMid:14571191. https://doi.org/10.1097/00002030200311070-00014

31. Gallego O, Ruíz L, Vallejo A, Clotet B, Leal M, Soriano V. Rate of virological treatment failure and frequencies of drug resistance genotypes among human immunodeficiency virus-positive subjects on antiretroviral therapy in Spain. J Clin Microbiol. 2002;40(10):3865-3866. PMid:12354903 PMCid:PMC130857.https://doi.org/10.1128/jcm.40.1 $0.3865-3866.2002$

32. Costagliola D, Descamps D, Assoumou L, et al. Prevalence of HIV-1 drug resistance in treated patients: a French nationwide study. J Acquir Immune Defic Syndr. 2007;46(1):12-18. PMid:17514016. https://doi.org/10.1097/QAl.0b013e318074eb73

33. Scott P, Arnold E, Evans B, et al. Surveillance of HIV antiretroviral drug resistance in treated individuals in England: 1998-2000. J Antimicrob Chemother. 2004;53(3):469-473. https://doi.org/10.1093/jac/dkh102

34. El Annaz H, Recordon-Pinson P, Tagajdid R, et al. Drug resistance mutations in HIV type 1 isolates from patients failing antiretroviral therapy in Morocco. AIDS Res Hum Retroviruses. 2012;28(8):944-948. 
PMid:21919803

PMCid:PMC3399567. https://doi.org/10.1089/AID.2011.0278

35. Sukasem C, Churdboonchart V, Chasombat S, et al. Prevalence of antiretroviral drug resistance in treated HIV-1 infected patients: under the initiative of access to the NNRTI-based regimen in Thailand. J Chemother. 2007;19(5):528-535. PMid:18073152. https://doi.org/10.1179/joc.2007.19.5.528

36. Hosseinipour MC, van Oosterhout JJ, Weigel R, et al. The public health approach to identify antiretroviral therapy failure: high-level nucleoside reverse transcriptase inhibitor resistance among Malawians failing first-line antiretroviral therapy. AIDS. 2009;23(9):1127-1134. PMid:19417582 PMCid:PMC2896488.https://doi.org/10.1097/QAD.0b 013e32832ac34e

37. Gupta R, Hill A, Sawyer AW, Pillay D. Emergence of drug resistance in HIV type 1-infected patients after receipt of first-line highly active antiretroviral therapy: a systematic review of clinical trials. Clin Infect Dis. 2008;47(5):712-722. PMid:18662137. https://doi.org/10.1086/590943

38. Fantini J, Tamalet C, Yahi N. Secondary structure predictions of HIV-1 reverse transcriptase provide new insights into the development of drug-resistance genotypes. AIDS. 2001;15(9):1191-1192. PMid:11416728. https://doi.org/10.1097/00002030200106150-00021

39. Saag MS, Benson CA, Gandhi RT, et al. Antiretroviral Drugs for Treatment and Prevention of HIV Infection in Adults: 2018 Recommendations of the International Antiviral Society-USA Panel. JAMA. 2018;320(4):379-396. PMid:30043070 PMCid:PMC6415748.https://doi.org/10.1001/jama.20 18.8431 